\title{
What is the Brightest Source for Dilepton Emissions at RHIC?
}

\author{
David Fein, Zheng Huang*, Peter Valerio and Ina Sarcevic \\ Department of Physics, University of Arizona, Tucson, AZ 85721, USA
}

(July, 1996)

\begin{abstract}
We calculate the dilepton emissions as the decay product of the charm and bottom quarks produced in heavy-ion collisions at RHIC energy. We take into account the next-to-leading-order radiative corrections in perturbative QCD to the heavy quark production from both an initial hard parton-parton scattering and an ideal quark-gluon plasma. We find that the thermal charm decay dominates the dilepton production in the low dilepton mass region $(<2$ $\mathrm{GeV}$ ), while the heavy quark production from the initial scattering takes over the intermediate and high mass regions $(>2 \mathrm{GeV})$. Our result also indicates the importance of the bottom quark in the high mass region $(>4 \mathrm{GeV})$ due to its large mass and cascade decay. If the initial scattering produced charm suffers a significant energy loss due to the secondary interaction, the bottom decay constitutes the major background for the thermal dileptons.
\end{abstract}

*Electronic address: huang@physics.arizona.edu 
The emission of dileptons in high energy heavy-ion collisions provides an excellent probe of the property of dense hadronic matter such as the quark-gluon plasma and the hot hadronic gas. Due to the small cross sections of electromagnetic interactions, these dileptons once produced can escape the strong interacting volume of sizes which might be produced in heavy-ion collisions. The dilepton invariant mass spectrum holds the most promising signatures of the quark-gluon plasma such as thermal dileptons [1] and the $\mathrm{J} / \psi$ suppression [2]. However, the use of the dilepton probe is difficult for an obvious reason: it is sensitive to many different sources. In the low mass region, the resonance decays from the light hadrons constitute the main background. In the intermediate and high mass regions, the Drell-Yan dilepton production from the initial hard scattering is important. In high energy heavy-ion colliders such as RHIC and LHC, the heavy flavor quark production can be quite substantial and its subsequent decay can lead to a large combinatorial background for the dilepton spectrum. Therefore it is crucial to make a solid theoretical prediction on the contribution from heavy quark decays.

In the Letter we discuss the dimuon emission as the decay product of the heavy quarks (charm and bottom) produced in an initial hard scattering and in a thermalized quarkgluon plasma at RHIC energy. We include the next-to-leading order radiative corrections and nuclear shadowing effects. Indeed, the recent work by Gavin, McGaughey, Russkanen and Vogt [3] has indicated that the dilepton production from the correlated charm decay is by far the dominant contribution. However, if the energetic charmed parton is subject to energy losses due to multiple secondary interactions in the dense matter produced in the collisions, the dilepton spectrum may be considerably softened, as suggested recently by Shuryak [ []. In contrast to the charm, the bottom decay can yield higher invariant mass lepton pairs even at rest due to the large bottom mass. It is thus necessary to examine the various components of the heavy quark spectra and their role in the dilepton production in an extended dilepton mass region. We also examine the sensitivities of the perturbative calculation on the choices of the relevant physical scale and the heavy quark fragmentation function. The heavy parton production is calculated using the explicit next-to-leading order 
matrix elements, while the parton fragmentation and the heavy hadron decay are handled by the IsAJET Monte Carlo model [5]. The cascade decay of the bottom quark, $b \rightarrow c \rightarrow s$ is also included in this model.

Theoretical calculations of heavy quark differential and total cross sections to the nextto-leading order $O\left(\alpha_{s}^{3}\right)$ at RHIC and LHC energies have been performed by several groups [6] for the initial hard scatterings. It is found that higher order radiative corrections are important and the theoretical $K$-factor ranging from $1 \sim 3$ has strong transverse momentum dependence. The nuclear shadowing effect is also non-negligible even at RHIC energy. Therefore, it is crucial to include full next-to-leading order corrections when one evaluates the dilepton production from heavy-quark decay.

The production of heavy hadrons is based on the factorization theorem, which states that the cross section for the production process of a hadron with an energy-momentum $(E, \mathbf{k})$ can be written in a factorized form

$$
E \frac{d \sigma}{d^{3} k}=\sum_{i} \int E^{\prime} \frac{d \sigma_{i}}{d^{3} k^{\prime}}\left(k^{\prime}, \mu\right) D_{i}^{(h)}(z, \mu) \frac{E}{E^{\prime}} \frac{d z}{z^{3}}
$$

where $k^{\prime}=k / z, D_{i}^{(h)}(z, \mu)$ is the fragmentation function for producing the hadron $h$ from the parton $i$ and $d \sigma_{i}$ is the short-distance cross section for producing the parton $i$ from the colliding hadrons. Clearly if $i$ is a light parton, the cross section $d \sigma_{i}$ is not finite and the prescription for subtracting the collinear singularity has to be employed. However, in the case of heavy quark production, $d \sigma_{i}$ is an infrared safe quantity because the heavy quark mass acts as a cutoff for final state collinear singularities. Thus, the heavy quark mass sets the scale for a perturbative expansion in the strong coupling constant. In the limit when the mass can be considered much larger than the typical hadronic scale, the fragmentation function $D_{i}^{(h)}(z, \mu)$ is also calculable in perturbation theory. As long as the transverse momentum of the produced heavy quark is not much larger than its mass, perturbation theory predicts $D_{i}^{(h)}(z) \sim \delta(1-z)$. On the other hand, the finite mass and energy transfer $(\Delta E)$ in the fragmentation process suggests that the breakup amplitude $\sim 1 / \Delta E$, where 


$$
\begin{aligned}
\Delta E & =\left(m_{Q}^{2}+z^{2} k^{2}\right)^{1 / 2}+\left(m_{q}^{2}+(1-z)^{2} k^{2}\right)^{1 / 2}-\left(m_{Q}^{2}+k^{2}\right)^{1 / 2} \\
& \propto 1-1 / z-\epsilon_{Q} /(1-z) .
\end{aligned}
$$

This leads to a Peterson form [7]

$$
D_{Q}^{(H)}=\frac{N}{z\left[1-(1 / z)-\epsilon_{Q} /(1-z)\right]^{2}},
$$

where $\epsilon_{c}=0.15$ and $\epsilon_{b}=0.016$. The normalization constant $N$ is fixed by the sum rule $\int d z D_{Q}^{H}(z)=1: \quad N=1.28,0.25$ for $c$ and $b$ respectively. Such a parameterization has been shown to be appropriate in $e^{+} e^{-}$reactions and fit well the MARK-II data [0]. For hadronic collisions or an even heavier quark decay, it is known that there are no universal fragmentation functions. The double differential cross sections for (all) charmed hadrons (mainly $D$-mesons) as well as (all) $b$-hadrons (mainly $B$-mesons) are calculated using Eq.(11) (assuming that there is no energy loss for the produced parton before hadronization) and the results are plotted in Fig.1 against the distributions for the heavy partons.

The initial parton differential distributions are calculated using the next-to-leading-order $(\mathrm{NLO})$ matrix elements provided by Nason, Dawson and Ellis in [8]. We have taken $m_{c}=1.5$ $\mathrm{GeV}, m_{b}=4.7 \mathrm{GeV}$, the factorization and renormalization scale $\mu$ to be the transverse mass $2 m_{t}$. The nucleon structure function is parameterized by MRS A and the nuclear shadowing parametrization fits the EMC, NMC and E665 data [9]. The total cross sections (per initial nucleon) are $\sigma_{c \bar{c}}^{\text {tot }}=153 \mu \mathrm{b}$ and $\sigma_{b \bar{b}}^{\text {tot }}=1.5 \mu \mathrm{b}$ for $\mathrm{Au}+\mathrm{Au}$ collisions at $\sqrt{s}=200 \mathrm{GeV} / \mathrm{n}$. The choice of a smaller charm quark mass $m_{c}=1.2 \mathrm{GeV}$ increases the total cross section by a factor of 2 (see Vogt in [6]). 


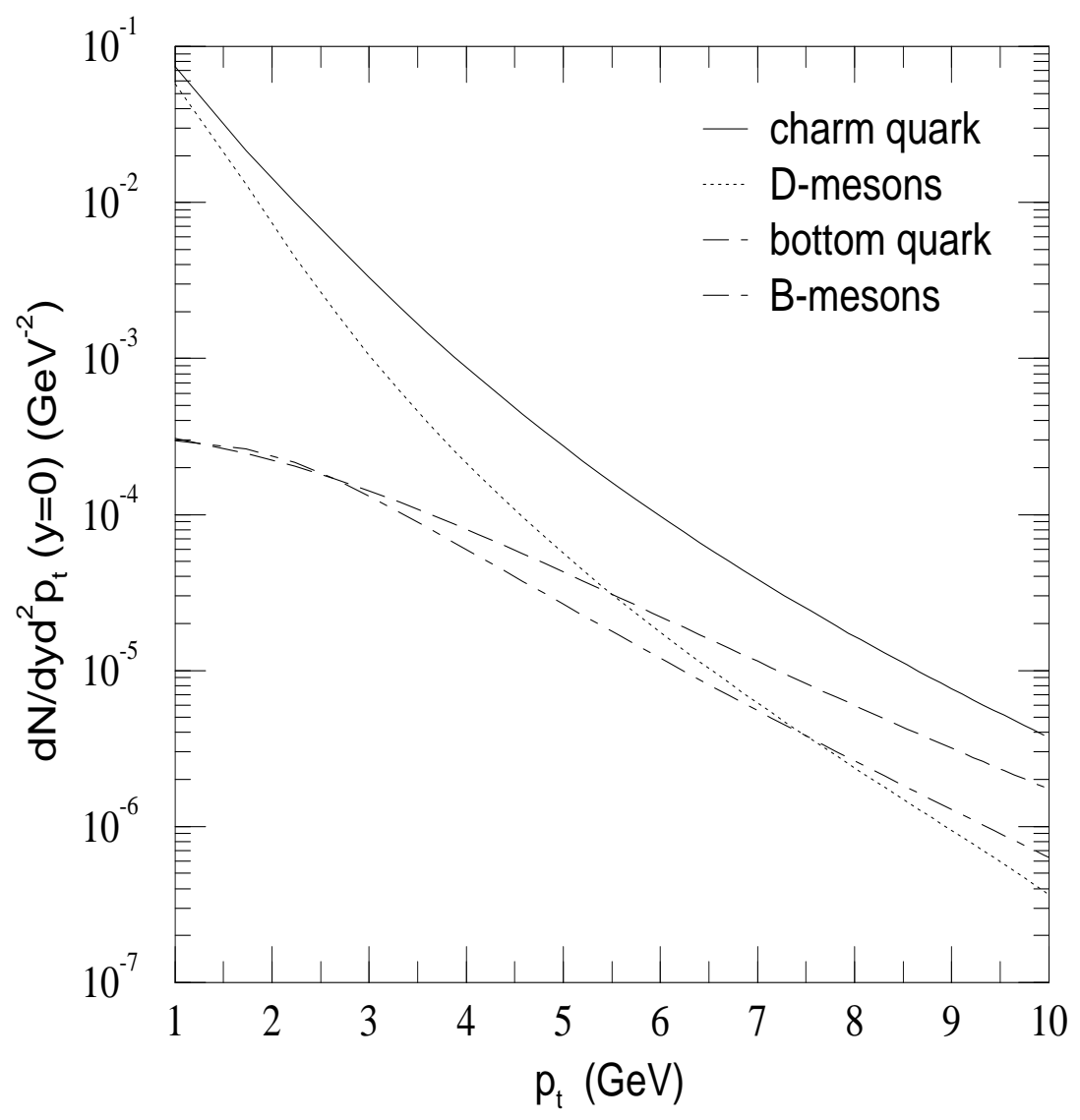

FIG. 1. The heavy quark hadron differential cross sections calculated using the Peterson heavy quark fragmentation functions convoluted with the parton cross sections. The initial heavy quark productions are calculated using the explicit next-to-leading-order matrix elements with MRS A structure function and the nuclear shadowing function parameterized by Benesh et. al. [9]

As is evident from Fig.1, the leading order (in heavy quark mass) fragmentation function $D(z) \sim \delta(1-z)$ seems to be a reasonable approximation for heavy quarks. There is some noticeable softening of heavy mesons at large $p_{t}$ and an enhancement of $B$-meson at very small $p_{t}$ due to the finite heavy quark mass and the heavy quark-hadron mass splitting.

The semileptonic decay of the heavy meson can be conveniently handled by Monte Carlo simulations such as IsAJET or Jetset. These Monte Carlo programs usually have the leading order matrix elements as the default value while the initial and final state radiations are treated by a parton shower model. The virtue of the parton shower algorithm is that 
it is beyond the leading logarithmic approximation and incorporates the multiple parton emissions. On the other hand, it is not a consistent higher order calculation since it does not contain the virtual corrections in the matrix elements. Simply embedding the NLO matrix elements in the Monte Carlo program may cause an ambiguity due to double counting of multiple parton emissions. To circumvent this difficulty and to preserve the NLO heavy quark production calculation, we develop a numerical routine to generate the events at the parton level. We explicitly integrate out the initial parton momenta in the total cross section Monte Carlo integral and write out the four-momentum for each heavy quark. These momenta are associated with the corresponding weight in the phase space multiplied by the corresponding value of the transition probability. Summing up these weights gives the total cross section. One can then use IsAJET to fragment each parton pair and to track the subsequent correlated decays.

When generating events, one encounters a genuine feature of the higher-order calculation: the presence of the terms needed to cancel the collinear singularities in the initial state radiation. In the factorization scheme, one uses the "+ function" prescription to calculate these terms. As a result, the events associated with the substraction terms have to be generated in the Monte Carlo integration of the matrix elements. There is no guarantee that the differential cross section should always add up to a positive value everywhere in the presence of the substraction terms. Some negative bins may appear in the region close to the boundary of phase space for real emission. The problem is worse when one calculates the dilepton spectrum. Due to the statistical nature of the decay process, there is no prediction where the negative bins may appear in the dilepton invariant mass spectrum. In order to make the distribution more readable in the presence of such negative bins, we will always enlarge the size of the these bins so as to integrate over more and more positive real contributions.

At sufficiently high temperatures, a thermal system of light partons can create a charm pair which will decay into leptons and should also be included. The thermal charm production has been calculated so far at the leading order matrix element level [10]. To estimate 
the reliability of the leading-order result, one has to perform a full NLO calculation. At the next-to-leading order, there are additional $2 \rightarrow 3$ processes and loop diagrams. Formally, the differential production rate is given by the convolution of the parton level differential cross sections with the initial light parton phase space densities (distribution functions) $f_{i}(p)$ (we neglect the final state Fermi blocking or Bose-Einstein enhancement )

$$
\begin{aligned}
E \frac{d R}{d^{3} k}= & \int \frac{d^{3} p_{1}}{(2 \pi)^{3} 2 E_{1}} \frac{d^{3} p_{2}}{(2 \pi)^{3} 2 E_{2}} F\left(p_{1}, p_{1}\right)\left[\frac{g_{g g}}{2} f_{g}\left(p_{1}\right) f_{g}\left(p_{2}\right) E \frac{d \hat{\sigma}_{g g}}{d^{3} k}+\right. \\
& \left.g_{q \bar{q}} f_{q}\left(p_{1}\right) f_{\bar{q}}\left(p_{2}\right) E \frac{d \hat{\sigma}_{q \bar{q}}}{d^{3} k}+g_{q g} f_{q}\left(p_{1}\right) f_{g}\left(p_{2}\right) E \frac{d \hat{\sigma}_{q g}}{d^{3} k}\right]
\end{aligned}
$$

where $g_{g g}=16^{2}, g_{q \bar{q}}=6^{2} N_{f}$ and $g_{q g}=6 \cdot 16 N_{f}$ are the degeneracy factors, and $F\left(p_{1}, p_{2}\right)=$ $4 p_{1} p_{2}$ is the inverse flux factor.

At the leading order, the $d \hat{\sigma}_{q g}$ term is absent. At the next-to-leading order, these parton level differential cross sections contain $2 \rightarrow 3$ processes and loop diagrams and they are not infrared finite quantities. Braaten and Pisarski [11] have shown that a resummation technique has to be employed to take into account the so-called "hard thermal loops" which give the same order of magnitude contributions as the leading order terms when the external momenta of the amplitude are soft. As a result of the resummation, the infrared singularities are shielded by a Debye screening effect, and the screening masses of gluons and quarks provide an infrared cutoff. The perturbative resummation can only regularize the electric part of the propagators and so far the magnetic screening mass has to come from the unknown nonperturbative effect. To obtain a qualitative result for the NLO thermal charm production, we use the Debye screening mass 12

$$
\mu_{D}^{2}=\frac{6 g^{2}}{\pi^{2}} \int p f(p) d p=4 \pi \alpha_{s} T^{2}
$$

to regularize all singularities in the radiative cross sections. This amounts to a "massive

\footnotetext{
1 When the final state quantum statistical effect is present, one also needs to consider $3 \rightarrow 2$ processes in order to organize the collinear singularity in a familiar factorizable form. We do not consider this possibility.
} 
gluon scheme" where the $\mu_{D}^{2}$ sets the scale for the parton level cross sections in Eq.(四). Certainly, this calculation is not entirely consistent, and the results thus obtained can only be taken as an qualitative indication on the reliability of the leading-order result.

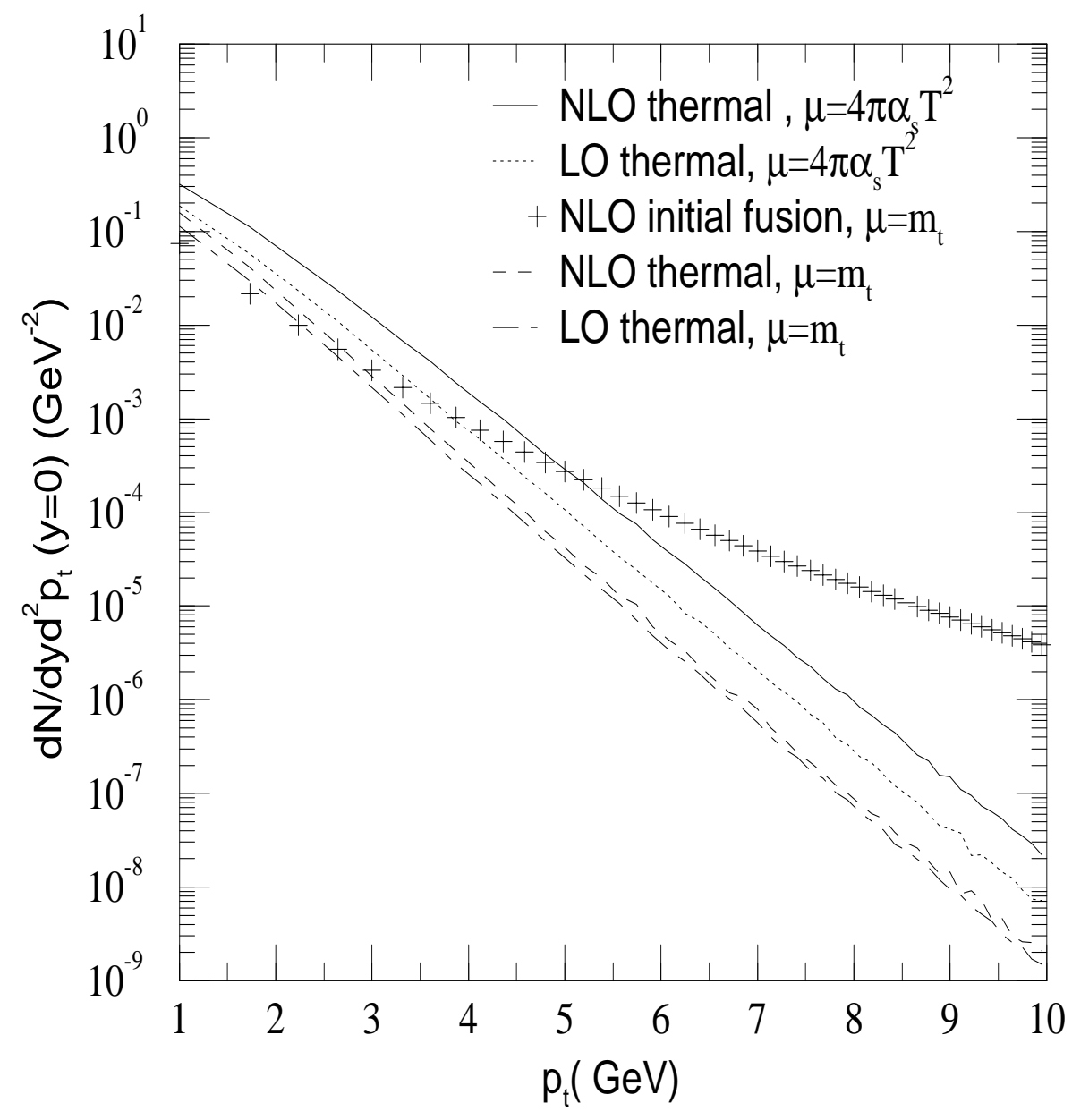

FIG. 2. The charm differential distribution produced in an ideal thermalized quark-gluon plasma at the leading and the next-to-leading orders in perturbative QCD. The sensitivity on the choice of the scale is illustrated. The charm production at the NLO from the initial hard scattering is also plotted for comparison.

We have evaluated the thermal charm yield at RHIC energy by integrating over the spacetime evolution of the quark-gluon plasma. We assume a fully equilibrated thermal system with an initial temperature $T_{0}=0.55 \mathrm{GeV}$ and a Bjorken ideal-fluid scaling $T(\tau) / T_{0}=$ 
$\left(\tau_{0} / \tau\right)^{1 / 3}$ with $\tau_{0}=0.7 \mathrm{fm} / \mathrm{c}$ and $T_{c}=0.2 \mathrm{GeV}$. A spatial rapidity cutoff $\eta_{\max }=3$ is assumed. The differential distributions are plotted in Fig.2 for the leading order and the next-to-leading order calculations using the Debye screening scale in (5). We also compare the result for a choice of the scale $\mu=m_{t}$. As in the initial hard scattering case, the higher order corrections are sizable with an effective $K$-factor ranging from 1.7 to 2.5 as $p_{t}$ varies from $1 \mathrm{GeV}$ to $10 \mathrm{GeV}$. We use the leading order kinematics to generate the heavy quark momenta and multiply the weights uniformly by an average value $K=2$ to account for the radiative corrections.

The calculated dimuon spectra from the decay of heavy quarks produced in a hard scattering and in an idealized quark-gluon plasma are plotted in Fig.3. The dimuon spectrum from thermal charm quark production is considerably softer than the initial hard scattering results as can be inferred from the $p_{t}$ distribution in Fig.2. However, it dominates the dilepton production in the low mass region $(M<2 \mathrm{GeV})$. This can be a important background at RHIC for the dilepton signature for the low lying vector resonances such as $\rho$ and $\phi$. It is important to note that the calculated thermal charm contribution corresponds to a very ideal case where the quarks and gluons are fully equilibrated. The result may be considerably smaller in the plasma where the chemical process is not in equilibrium [10]. The thermal production of the bottom quark is negligible and is not included in Fig.3. The cascade decay of $B$-mesons, $b \rightarrow c \rightarrow s$ is also included in the curve for the initial fusion bottom production. We have not assumed any secondary interactions in the medium produced in the collision. Although the production rate for the initial bottom quark is much smaller than the charm, the dilepton production from $B$-meson decay is non-negligible in the large invariant mass region due to the large rest mass of the $B$-meson?. It becomes the dominant contribution when the dimuon invariant mass is above $\sim 4 \mathrm{GeV}$. If the fast

\footnotetext{
2 The results in [3] give a larger initial charm contribution due to the choice of a smaller charm mass. However, we do not have a complete understanding of their rather small bottom contribution.
} 
charm suffers the significant energy loss before it hadronizes, the dimuon spectrum from the initial fusion charm can be considerably softened. In fact, it becomes very similar to that of the thermal charm and falls off rapidily as $M$ gets bigger than $2 \mathrm{GeV}$, as demonstrated by Shuryak [1]. In this case, the bottom dominance is more prominent and starts at a smaller value of $M \sim 2 \mathrm{GeV}$ because two $B$-mesons at rest can produce an energetic lepton pair. To make a comparison, the Drell-Yan dimuon production taken from [3] is also indicated in the plot.

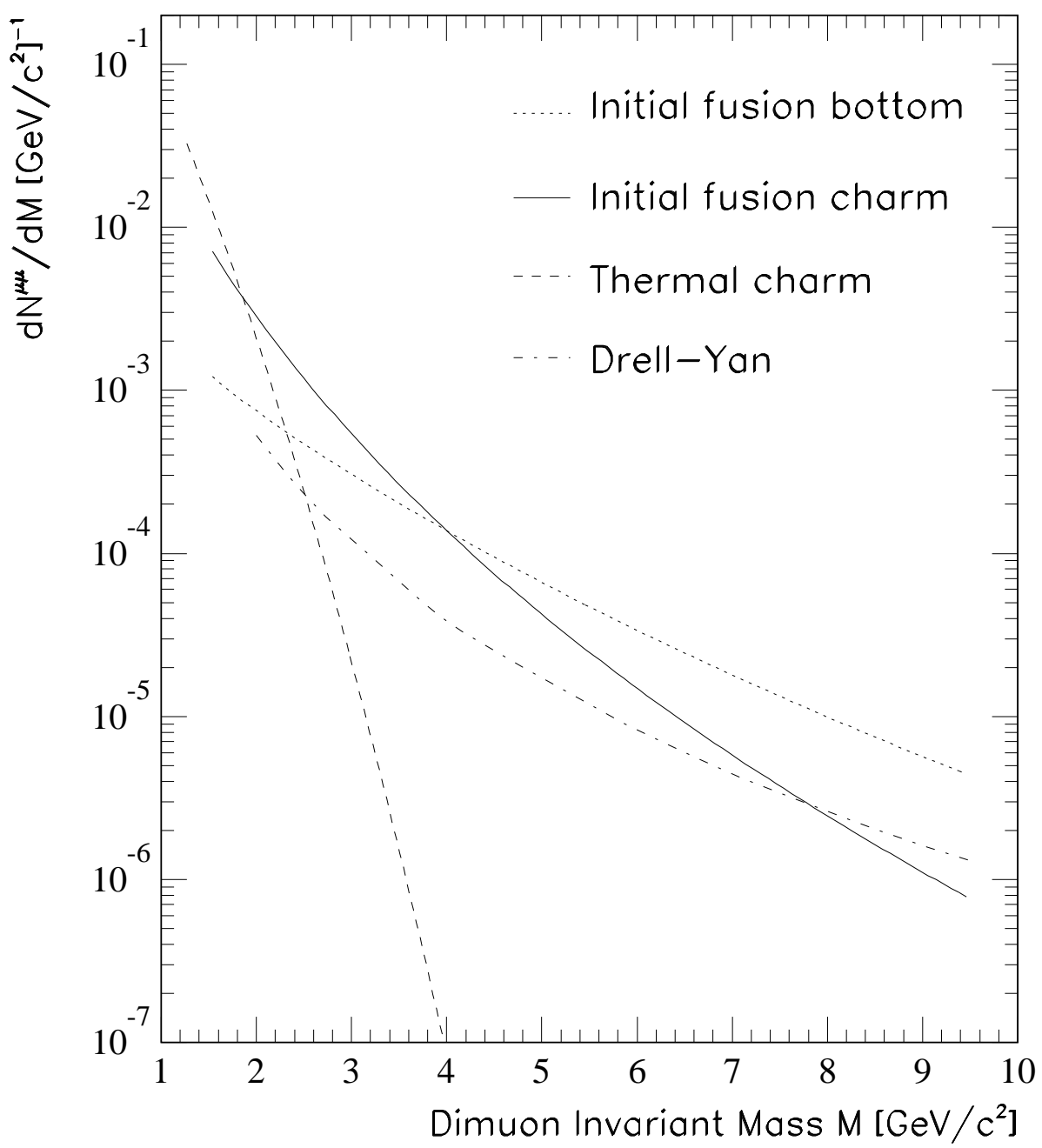

FIG. 3. The dimuon invariant mass spectra from the correlated heavy quark decay calculated using IsAJET. No energy loss for the fast parton is assumed. 
In conclusion, we have evaluated the dilepton emissions from the decay of the heavy quark productions from the initial hard scatterings and from a quark-gluon plasma in $\mathrm{Au}+\mathrm{Au}$ collisions at RHIC energy $\sqrt{s}=200 \mathrm{GeV} / \mathrm{n}$. We check the reliability of the leading order results by explicitly performing a next-to-leading-order calculation. The thermal charm from an ideal quark-gluon plasma dominates the dilepton emission in the low dilepton invariant mass region $(<2 \mathrm{GeV})$. The correlated heavy mesons produced from the initial hard scattering constitutes the major contribution to the intermediate and high mass dileptons. If there is significant energy loss for the fast charm, the bottom decay is by far the dominant component in the intermediate and high dimuon mass regions. In any case, we are likely to find more bottom than charm in the high mass dilepton channel in central $\mathrm{Au}+\mathrm{Au}$ collisions at RHIC.

We would like to thank R. Vogt for useful discussions. Z.H. and I.S. Thank the Aspen Center for Physics for hospitality where part of this work is accomplished. This work was supported in part by the U.S. Department of Energy Contract No. DE-FG03-93ER40792. 


\section{REFERENCES}

[1] E. Shuryak, Phys. Lett. B78, 150 (1978)

[2] T. Matsui and H. Satz, Phys. Lett. B178, 416 (1986)

[3] S. Gavin, P.L. McGaughey, P.V. Ruuskanen and R. Vogt, LBL Preprint LBL-37981 (1996)

[4] E. Shuryak, nucl-th/9605011 (1996).

[5] F.E. Paige, S.D. Protopopescu, Snowmass Summer Study 1986:320

[6] I. Sarcevic and P. Valerio, Phys. Lett. B338, 426 (1994); Phys. Rev. C51, 1433 (1995); R. Vogt, LBL-37105 (1995).

[7] C. Peterson, D. Schlatter, I. Schmitt and P.M. Zerwas, Phys. Rev. D27, 105 (1983)

[8] P. Nason, S. Dawson and R.K. Ellis, Nucl. Phys. B327, 49 (1989); B303, 607 (1988);

M.L. Mangano, P. Nason and G. Ridolfi, Nucl. Phys. B373, 295 (1992)

[9] C.J. Benesh, J. Qiu and J.P. Vary, Phys. Rev. C50, 1015 (1994)

[10] P. Levai, B. Müller and X.-N. Wang, Phys. Rev. C51, 3326 (1995); Z. Lin and M. Gyulassy, Phys. Rev. C51, 2177 (1995)

[11] E. Braaten and R.D. Pisarski,Nucl. Phys. B337, 569 (1990)

[12] T.S. Biro, B. Müller and X.-N. Wang, Phys. Lett. B283, 171 (1992) 